\title{
q-Gamow states for intermediate energies
}

\author{
A. Plastino ${ }^{\text {a }}$, M.C. Rocca ${ }^{\text {a,* }}$, G.L. Ferri ${ }^{\text {b }}$, D.J. Zamora ${ }^{\text {a }}$ \\ ${ }^{a}$ La Plata National University and Argentina's National Research Council, (IFLP-CCT-CONICET)-C. C. 727, \\ 1900 La Plata, Argentina \\ ${ }^{\mathrm{b}}$ Fac. de C. Exactas, National University La Pampa, Peru y Uruguay, Santa Rosa, La Pampa, Argentina
}

Received 23 April 2016; received in revised form 22 May 2016; accepted 27 May 2016

Available online 2 June 2016

\begin{abstract}
In a recent paper Plastino and Rocca (2016) [18] we have demonstrated the possible existence of Tsallis' q-Gamow states. Now, accelerators' experimental evidence for Tsallis' distributions has been ascertained only at very high energies. Here, instead, we develop a different set of q-Gamow states for which the associated q-Breit-Wigner distribution could easily be found at intermediate energies, for which accelerators are available at many locations. In this context, it should be strongly emphasized Vignat and Plastino (2009) [2] that, empirically, one never exactly and unambiguously "detects" pure Gaussians, but rather q-Gaussians. A prediction is made via Eq. (3.4).

(C) 2016 Elsevier B.V. All rights reserved.
\end{abstract}

Keywords: Gamow states; q-Gamow states; q-Gaussians

\section{Introduction}

Empirical analysis abundantly shows that power-law behavior in the (observed) distribution of some quantity is rather common in nature [1]. It has been shown that a reason for this high frequency is detector-normalization [2]. Since very many systems are statistically described by power-law probability distributions [3], this is a subject that deserves attention. In particular, q-Gaussian behavior is frequently found in different scenarios. Reference [2] explains why. This happens in experimental scenarios for which data are gathered using a set-up that performs a

\footnotetext{
* Corresponding author.

E-mail address: mariocarlosrocca@gmail.com (M.C. Rocca).
} 
normalization-preprocessing. [2] finds that in such settings the value of the associated parameter $\mathrm{q}$ can be deduced from the normalization technique that characterizes the empirical device. Of course, by a q-exponential we mean the function

$$
e_{q}(x)=[1+(1-q) x]^{1 /(1-q)},
$$

that tends to the ordinary exponential when $q$ approaches unity [4].

It was shown in [5-8] that resonances, i.e. Gamow-states [9,10], can be seen as (Sebastiao e Silva's) Ultradistributions [11-13]. Their treatment needs appealing to Rigged Hilbert Space [14-17]. It was demonstrated in [18] that associated resonances, called q-Gamow states, constitute a useful generalization of the GS concept adapted to Tsallis' q-statistics [4].

Indeed, one can find a large number of high energy experiments amenable to interpretation via Tsallis' q-statistics [4], specifically, LHC experiments in what concerns distributions connected to stationary states. Tsallis' q-statistics adequately describes the transverse momentum distributions of variegated hadrons. All four LHC experiments generated papers using such distributions that can be adequately fitted employing the q-exponential function. The pertinent q-value is of the order of 1.15, clearly distinct from the unity value of Gibbs-Boltzmann's statistics. This evidences that stationary states before hadronization can not be thermal equilibrium-ones [4]. Measurement of the $p_{\mathrm{T}}$ distribution over a logarithmic range of fourteen decades demonstrates that $q=1.15$ fits nicely the data over this large range $[19,20])$.

These findings motivate one to study complex energy states related to the q-exponential distributions (namely, q-Gamow states), that are not solutions of Schroedinger's equation but of its nonlinear, q-version counterpart, advanced Nobre, Rego-Monteiro, and Tsallis in [21] (see also [22]). We recommend, for a review on ordinary Gamow states, reference [23].

Remark that accelerators' experimental evidence for Tsallis' distributions has been ascertained only at very high energies. This motivates us to inquire about other kind of q-Gamow states for which the associated q-Breit-Wigner distribution could easily be found at intermediate energies, for which accelerators are available at many locations. Such is then the goal we seek to achieve here. WE base our considerations on the fact that, empirically, one does not often detect pure Gaussians, but rather q-Gaussians [2]. This suggests looking for special q-Gamow states in the q-neighborhood of $\mathrm{q}=1$.

\section{New q-Gamow states to be introduced here}

We obtain a new kind of Gamow state for q close to unity, via perturbation theory around such q-value, of the states studied in [18], keeping up to first order terms. Accordingly,

$$
\left[1+\frac{i(1-q) p x}{\hbar \sqrt{2(q+1)}}\right]^{\frac{2}{1-q}} \simeq\left[1-(q-1)\left(\frac{i p x}{4 \hbar}+\frac{p^{2} x^{2}}{4 \hbar^{2}}\right)\right] e^{\frac{i p x}{\hbar}}
$$

and the ensuing new q-Gamow state becomes

$$
\begin{aligned}
& \left|\psi_{\mathrm{qG}}\right\rangle=\int_{-\infty}^{\infty}\{\mathcal{H}[\Im(\mathrm{p})] \mathcal{H}(\mathrm{x})-\mathcal{H}[-\mathfrak{I}(\mathrm{p})] \mathcal{H}(-\mathrm{x})\} \\
& \otimes\left[1-(q-1)\left(\frac{i p x}{4 \hbar}+\frac{p^{2} x^{2}}{4 \hbar^{2}}\right)\right] e^{\frac{i p x}{\hbar}} \mid x>d x,
\end{aligned}
$$


or

$$
\begin{aligned}
\psi_{\mathrm{qG}}(x)= & \{\mathcal{H}[\Im(p)] \mathcal{H}(x)-\mathcal{H}[-\Im(p)] \mathcal{H}(-x)\} \\
& \otimes\left[1-(q-1)\left(\frac{i p x}{4 \hbar}+\frac{p^{2} x^{2}}{4 \hbar^{2}}\right)\right] e^{\frac{i p x}{\hbar}} .
\end{aligned}
$$

The norm of a q-Gamow state is now

$$
\begin{aligned}
\left\langle\psi_{\mathrm{qG}} \mid \psi_{\mathrm{qG}}\right\rangle= & \int_{0}^{\infty} \mathcal{H}[\Im(p)]\left[1-(q-1)\left(\frac{i p x}{4 \hbar}+\frac{p^{2} x^{2}}{4 \hbar^{2}}\right)\right] e^{\frac{i p x}{\hbar}} \\
& \otimes\left[1+(q-1)\left(\frac{i p^{*} x}{4 \hbar}-\frac{p^{* 2} x^{2}}{4 \hbar^{2}}\right)\right] e^{-\frac{i p^{*} x}{\hbar}} d x \\
& +\int_{-\infty}^{0} \mathcal{H}[-\Im(p)]\left[1-(q-1)\left(\frac{i p x}{4 \hbar}+\frac{p^{2} x^{2}}{4 \hbar^{2}}\right)\right] e^{\frac{i p x}{\hbar}} \\
\otimes & {\left[1+(q-1)\left(\frac{i p^{*} x}{4 \hbar}-\frac{p^{* 2} x^{2}}{4 \hbar^{2}}\right)\right] e^{-\frac{i p^{*} x}{\hbar}} d x, }
\end{aligned}
$$

or equivalently,

$$
\begin{aligned}
\left\langle\psi_{\mathrm{qG}} \mid \psi_{\mathrm{qG}}\right\rangle= & \int_{0}^{\infty} \mathcal{H}[\mathfrak{I}(p)]\left[1-(q-1)\left(\frac{i p x}{4 \hbar}+\frac{p^{2} x^{2}}{4 \hbar^{2}}\right)\right] e^{\frac{i p x}{\hbar}} \\
& \otimes\left[1+(q-1)\left(\frac{i p^{*} x}{4 \hbar}-\frac{p^{* 2} x^{2}}{4 \hbar^{2}}\right)\right] e^{-\frac{i p^{*} x}{\hbar}} d x \\
& +\int_{0}^{\infty} \mathcal{H}[-\mathfrak{I}(p)]\left[1+(q-1)\left(\frac{i p x}{4 \hbar}-\frac{p^{2} x^{2}}{4 \hbar^{2}}\right)\right] e^{-\frac{i p x}{\hbar}} \\
& \otimes\left[1-(q-1)\left(\frac{i p^{*} x}{4 \hbar}+\frac{p^{* 2} x^{2}}{4 \hbar^{2}}\right)\right] e^{\frac{i p^{*} x}{\hbar}} d x .
\end{aligned}
$$

After a little algebra, (2.5) becomes

$$
\begin{aligned}
\left\langle\psi_{\mathrm{qG}} \mid \psi_{\mathrm{qG}}\right\rangle= & \int_{0}^{\infty} \mathcal{H}[\Im(p)]\left\{1+(q-1)\left[\frac{i\left(p^{*}-p\right) x}{4 \hbar}-\frac{\left(p^{2}+p^{* 2}\right) x^{2}}{4 \hbar^{2}}\right]\right\} e^{\frac{i\left(p-p^{*}\right) x}{\hbar}} d x \\
& +\int_{0}^{\infty} \mathcal{H}[-\mathfrak{I}(p)]\left\{1+(q-1)\left[\frac{i\left(p-p^{*}\right) x}{4 \hbar}-\frac{\left(p^{2}+p^{* 2}\right) x^{2}}{4 \hbar^{2}}\right]\right\} \\
& \times e^{\frac{i\left(p^{*}-p\right) x}{\hbar}} d x .
\end{aligned}
$$

Integration is straightforward:

$$
\left\langle\psi_{\mathrm{qG}} \mid \psi_{\mathrm{qG}}\right\rangle=\frac{\hbar}{2|\mathfrak{I}(\mathrm{p})|}\left\{1+\frac{(\mathrm{q}-1)}{4}\left[1+\frac{\mathfrak{I}(p)^{2}-\mathfrak{R}(p)^{2}}{\mathfrak{I}(p)^{2}}\right]\right\} .
$$


Thus, squaring the norm we find

$$
A^{2}(q, p)=\frac{\hbar}{2|\mathfrak{I}(p)|}\left\{1+\frac{(q-1)}{4}\left[1+\frac{\mathfrak{I}(p)^{2}-\mathfrak{R}(p)^{2}}{\mathfrak{I}(p)^{2}}\right]\right\},
$$

and then one has

$$
A(q, p)=\sqrt{\frac{\hbar}{2|\mathfrak{I}(p)|}\left\{1+\frac{(q-1)}{4}\left[1+\frac{\mathfrak{I}(p)^{2}-\mathfrak{R}(p)^{2}}{\mathfrak{I}(p)^{2}}\right]\right\}} .
$$

Note that:

$$
\lim _{q \rightarrow 1} A(q, p)=\sqrt{\frac{\hbar}{2|\mathfrak{I}(p)|}}
$$

We see that (2.10) and (A.3) agree. The normalized q-Gamow state is now

$$
\left|\phi_{\mathrm{qG}}\right\rangle=\frac{\left|\psi_{\mathrm{qG}}\right\rangle}{\mathrm{A}(\mathrm{q}, \mathrm{p})},
$$

that can be recast in the fashion

$$
\begin{aligned}
\left|\phi_{\mathrm{qG}}\right\rangle= & \int_{-\infty}^{\infty}\{\mathcal{H}[\mathfrak{I}(p)] \mathcal{H}(x)-\mathcal{H}[-\mathfrak{I}(p)] \mathcal{H}(-x)\} \sqrt{\frac{2|\mathfrak{I}(\mathrm{p})|}{\hbar}} \\
& \otimes\left[1-(q-1)\left(\frac{1}{8}+\frac{\mathfrak{I}(p)^{2}-\mathfrak{R}(p)^{2}}{8 \mathfrak{I}(p)^{2}}+\frac{i p x}{4 \hbar}+\frac{p^{2} x^{2}}{4 \hbar^{2}}\right)\right] e^{\frac{i p x}{\hbar}}|d x\rangle,
\end{aligned}
$$

and thus

$$
\begin{aligned}
\phi_{\mathrm{qG}}(x)= & \{\mathcal{H}[\mathfrak{I}(\mathrm{p})] \mathcal{H}(x)-\mathcal{H}[-\mathfrak{I}(p)] \mathcal{H}(-x)\} \sqrt{\frac{2|\mathfrak{I}(\mathrm{p})|}{\hbar}} \\
& \otimes\left[1-(q-1)\left(\frac{1}{8}+\frac{\mathfrak{I}(p)^{2}-\mathfrak{R}(p)^{2}}{8 \mathfrak{I}(p)^{2}}+\frac{i p x}{4 \hbar}+\frac{p^{2} x^{2}}{4 \hbar^{2}}\right)\right] e^{\frac{i p x}{\hbar}} .
\end{aligned}
$$

We show now that the new q-Gamow states we are speaking about here satisfy the nonlinear q-Schroedinger equation of reference [22]. Consider the function $f(x)$ defined by

$$
f(x)=\sqrt{\frac{2|\mathfrak{I}(p)|}{\hbar}}\left[1-(q-1)\left(\frac{1}{8}+\frac{\mathfrak{I}(p)^{2}-\mathfrak{R}(p)^{2}}{8 \mathfrak{I}(p)^{2}}+\frac{i p x}{4 \hbar}+\frac{p^{2} x^{2}}{4 \hbar^{2}}\right)\right] e^{\frac{i p x}{\hbar}} .
$$

We wish to show that $f(x)$ satisfies

$$
H\left[\frac{f(x)}{f(0)}\right]=\frac{p^{2}}{2 m}\left[\frac{f(x)}{f(0)}\right]^{q}
$$

or

$$
H f(x)=\frac{p^{2}}{2 m}[f(0)]^{1-q}[f(x)]^{q},
$$

taking into account that 


$$
[f(0)]^{1-q}=1-(q-1) \ln \left[\sqrt{\frac{2|\mathfrak{I}(p)|}{\hbar}}\right],
$$

and

$$
\begin{aligned}
{[f(x)]^{q}=} & \sqrt{\frac{2|\mathfrak{I}(p)|}{\hbar}} e^{\frac{i p x}{\hbar}} \\
& \otimes\left\{1+(q-1)\left[\ln \left(\sqrt{\frac{2|\mathfrak{I}(p)|}{\hbar}}\right)-\frac{1}{8}-\frac{\mathfrak{I}(p)^{2}-\mathfrak{R}(p)^{2}}{8 \mathfrak{I}(p)^{2}}+\frac{3 i p x}{4 \hbar}-\frac{p^{2} x^{2}}{4 \hbar^{2}}\right]\right\} .
\end{aligned}
$$

Accordingly, we find

$$
\begin{aligned}
{[f(0)]^{1-q}[f(x)]^{q}=} & \sqrt{\frac{2|\mathfrak{I}(p)|}{\hbar} e^{\frac{i p x}{\hbar}}} \\
& \otimes\left\{1-(q-1)\left[\frac{1}{8}+\frac{\mathfrak{I}(p)^{2}-\mathfrak{R}(p)^{2}}{8 \mathfrak{I}(p)^{2}}-\frac{3 i p x}{4 \hbar}+\frac{p^{2} x^{2}}{4 \hbar^{2}}\right]\right\} \\
= & -\frac{1}{p^{2}} \frac{d^{2} f(x)}{d x^{2}}=\frac{2 m}{p^{2}} \operatorname{Hf}(x) .
\end{aligned}
$$

Minding (2.19) we see that $f(x)$ satisfies (2.15) and, consequently, the q-Gamow states also verify it.

We pass now to compute the mean value of the energy corresponding to a q-Gamow state. We begin with

$$
\begin{aligned}
\mathrm{H}\left|\phi_{\mathrm{qG}}\right\rangle= & \frac{\mathrm{p}^{2}}{2 \mathrm{~m}} \sqrt{\frac{2|\mathfrak{I}(\mathrm{p})|}{\hbar}} \\
& \otimes \int_{-\infty}^{\infty}\{\mathcal{H}[\mathfrak{I}(p)] \mathcal{H}(x)-\mathcal{H}[-\mathfrak{I}(p)] \mathcal{H}(-x)\} \\
& \otimes\left\{1-(q-1)\left[\frac{1}{8}+\frac{\mathfrak{I}(p)^{2}-\mathfrak{R}(p)^{2}}{8 \mathfrak{I}(p)^{2}}-\frac{3 i p x}{4 \hbar}+\frac{p^{2} x^{2}}{4 \hbar^{2}}\right]\right\} e^{\frac{i p x}{\hbar}} \mid x>d x,
\end{aligned}
$$

and thus

$$
\begin{aligned}
\left\langle\phi_{\mathrm{qG}}\left(\mathrm{H}\left|\phi_{\mathrm{qG}}\right\rangle\right)=\right. & \frac{p^{2}}{2 m} \frac{2|\mathfrak{I}(\mathrm{p})|}{\hbar} \otimes \int_{-\infty}^{\infty}\{\mathcal{H}[\mathfrak{I}(\mathrm{p})] \mathcal{H}(x)-\mathcal{H}[-\mathfrak{I}(p)] \mathcal{H}(-x)\} \\
& \otimes\left\{1-(\mathrm{q}-1)\left[\frac{1}{8}+\frac{\mathfrak{I}(p)^{2}-\mathfrak{R}(\mathrm{p})^{2}}{8 \mathfrak{I}(p)^{2}}-\frac{i p^{*} x}{4 \hbar}+\frac{p^{* 2} x^{2}}{4 \hbar^{2}}\right]\right\} \\
& \otimes\left\{1-(q-1)\left[\frac{1}{8}+\frac{\mathfrak{I}(p)^{2}-\mathfrak{R}(p)^{2}}{8 \mathfrak{I}(p)^{2}}-\frac{3 i p x}{4 \hbar}+\frac{p^{2} x^{2}}{4 \hbar^{2}}\right]\right\} \\
& \times e^{\frac{i\left(p-p^{*}\right) x}{\hbar}} \mathrm{d} x .
\end{aligned}
$$

The preceding equation can be recast as 


$$
\begin{aligned}
\left\langle\phi_{\mathrm{qG}}\left(\mathrm{H}\left|\phi_{\mathrm{qG}}\right\rangle\right)=\right. & \frac{p^{2}}{2 m} \frac{2|\mathfrak{I}(\mathrm{p})|}{\hbar} \otimes\left\{\int _ { 0 } ^ { \infty } \mathcal { H } [ \mathfrak { I } ( p ) ] \left\{1-(q-1)\left[\frac{1}{4}+\frac{\mathfrak{I}(p)^{2}-\mathfrak{R}(p)^{2}}{4 \mathfrak{I}(p)^{2}}\right.\right.\right. \\
& \left.\left.-\frac{i\left(3 p+p^{*}\right) x}{4 \hbar}+\frac{\left.\left(p^{2}+p^{* 2}\right) x^{2}\right]}{4 \hbar^{2}}\right]\right\} e^{\frac{i\left(p-p^{*}\right) x}{\hbar}} \mathrm{d} x \\
& \times \int_{0}^{\infty} \mathcal{H}[-\mathfrak{I}(p)]\left\{1-(q-1)\left[\frac{1}{4}+\frac{\mathfrak{I}(p)^{2}-\mathfrak{R}(p)^{2}}{4 \mathfrak{I}(p)^{2}}\right.\right. \\
& \left.\left.\left.+\frac{i\left(3 p+p^{*}\right) x}{4 \hbar}+\frac{\left(p^{2}+p^{* 2}\right) x^{2}}{4 \hbar^{2}}\right]\right\} e^{\frac{i\left(p^{*}-p\right) x}{\hbar}} \mathrm{dx}\right\} .
\end{aligned}
$$

Evaluating the integrals in (2.22) we encounter

$$
\left\langle\phi_{\mathrm{qG}}\right|\left(\mathrm{H}\left|\phi_{\mathrm{qG}}\right\rangle\right)=\frac{p^{2}}{2 m}\left\{1-(\mathrm{q}-1)\left[\frac{1}{4}-\frac{i\left(3 p+p^{*}\right)}{8|\mathfrak{I}(p)|} \operatorname{Sgn}[\Im(p)]\right]\right\} .
$$

Analogously, we reach

$$
\left(\left\langle\phi_{\mathrm{qG}}\right| \mathrm{H}\right)\left|\phi_{\mathrm{qG}}\right\rangle=\frac{p^{* 2}}{2 m}\left\{1-(q-1)\left[\frac{1}{4}-\frac{i\left(3 p^{*}+p\right)}{8|\mathfrak{I}(p)|} \operatorname{Sgn}[\Im(p)]\right]\right\} .
$$

Thus, according to [18], we obtain for the mean energy value

$$
\langle\mathrm{H}\rangle_{\mathrm{q}}=\frac{1}{2}\left[\left\langle\phi_{\mathrm{qG}}\right|\left(\mathrm{H}\left|\phi_{\mathrm{qG}}\right\rangle\right)+\left(\left\langle\phi_{\mathrm{qG}}\right| \mathrm{H}\right)\left|\phi_{\mathrm{qG}}\right\rangle\right] .
$$

Additionally, we have

$$
\lim _{\mathrm{q} \rightarrow 1}\langle\mathrm{H}\rangle_{\mathrm{q}}=\frac{\mathfrak{R}\left(\mathrm{p}^{2}\right)}{2 \mathrm{~m}}=\langle\mathrm{H}\rangle
$$

\section{Prediction: q-Breit-Wigner distribution}

We compute now the pertinent new q-Breit-Wigner distribution. We begin with

$$
\begin{aligned}
\left\langle\phi \mid \phi_{\mathrm{Gq}}\right\rangle= & \frac{1}{\hbar} \sqrt{\frac{|\mathfrak{I}(\mathrm{p})|}{\pi}}\left\{\int_{-\infty}^{\infty}\{\mathcal{H}[\mathfrak{I}(\mathrm{p})] \mathcal{H}(x)-\mathcal{H}[-\mathfrak{I}(\mathrm{p})] \mathcal{H}(-x)\}\right. \\
& \times\left[1-(\mathrm{q}-1)\left(\frac{1}{8}+\frac{\mathfrak{I}(p)^{2}-\mathfrak{R}(p)^{2}}{8 \mathfrak{I}(p)^{2}}+\frac{i p x}{4 \hbar}+\frac{p^{2} x^{2}}{4 \hbar^{2}}\right)\right] e^{\frac{i(p-k) x}{\hbar}} \mathrm{d} x .
\end{aligned}
$$

After evaluation the integrals in (3.1) we have

$$
\begin{aligned}
\left\langle\phi \mid \phi_{\mathrm{Gq}}\right\rangle= & \frac{1}{i(k-p)} \sqrt{\frac{|\mathfrak{I}(p)|}{\pi}} \\
& \otimes\left[1-(q-1)\left(\frac{1}{8}+\frac{\mathfrak{I}(p)^{2}-\mathfrak{R}(p)^{2}}{8 \mathfrak{I}(p)^{2}}+\frac{p}{4(k-p)}-\frac{p^{2}}{2(k-p)^{2}}\right)\right] .
\end{aligned}
$$

Thus, the q-Breit-Wigner relation is 


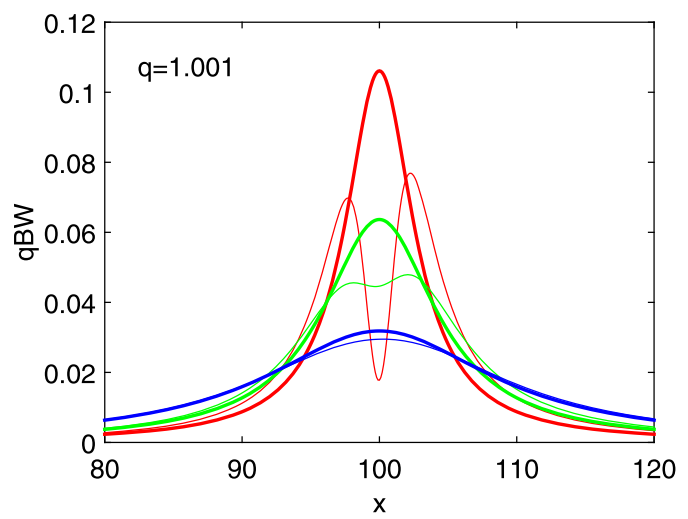

Fig. 1. $\mathrm{qBW}$ and orthodox BW vs. $x$, the real part of $p$, for $q=1+10^{-3}$. For additional details, see text. (For interpretation of the references to color in this figure, the reader is referred to the web version of this article.)

$$
\begin{aligned}
\left|\left\langle\phi \mid \phi_{\mathrm{Gq}}\right\rangle\right|^{2}= & \frac{|\mathfrak{I}(\mathrm{p})|}{\pi\left\{[\mathfrak{R}(\mathrm{p})-\mathrm{k}]^{2}+\mathfrak{I}(\mathrm{p})^{2}\right\}} \\
& \times\left\{1-(\mathrm{q}-1)\left[\frac{1}{4}+\frac{\mathfrak{I}(\mathrm{p})^{2}-\mathfrak{R}(\mathrm{p})^{2}}{4 \mathfrak{I}(\mathrm{p})^{2}}+\mathfrak{R}\left(\frac{\mathrm{p}}{2(\mathrm{k}-\mathrm{p})}\right)\right.\right. \\
& \left.\left.-\mathfrak{R}\left(\frac{\mathrm{p}^{2}}{(\mathrm{k}-\mathrm{p})^{2}}\right)\right]\right\} .
\end{aligned}
$$

The factor $X$

$$
X=(q-1)\left[\frac{1}{4}+\frac{\mathfrak{I}(p)^{2}-\mathfrak{R}(p)^{2}}{4 \mathfrak{I}(p)^{2}}+\mathfrak{R}\left(\frac{p}{2(k-p)}\right)-\mathfrak{R}\left(\frac{p^{2}}{(k-p)^{2}}\right)\right]
$$

constitutes the signature of our new q-Gamow resonances and is, in principle, amenable of empirical verification.

Note that for $q \rightarrow 1$ one has

$$
\lim _{q \rightarrow 1}\left|\left\langle\phi \mid \phi_{G q}\right\rangle\right|^{2}=\frac{|\mathfrak{I}(p)|}{\pi\left\{[\Re(p)-k]^{2}+\mathfrak{I}(p)^{2}\right\}}
$$

in agreement with (A.10).

We will make now what we believe to be a reasonable conjecture that relates q-values to energy ranges. We saw in [18] that finite-q's resonances take place at energies of the TeV scale for $q=1.1$. On the other hand, ordinary resonances appear at the $\mathrm{MeV}$ scale in BW descriptions $(q=1$ in our parlance). At this scale we also encounter quantum field theory resonances. By linear interpolation between these two instances we can guess that $q=1+10^{-3}$ should be associated to energies of about $1 \mathrm{GeV}, \mathrm{q}=1+10^{-4}$ to $0.1 \mathrm{GeV}$, etc.

Four cases of our small q's Breit-Wigner distribution (qBW)'s amplitudes (predictions) are illustrated in Figs. 1-4, for four q-values equal, respectively, to $1+10^{-3}-1+10^{-6}$, in steps of $10^{-1}$ added to unity. These q-values correspond, respectively, to energies ranging down from the $\mathrm{GeV}$ scale (associated to $\mathrm{q}=1+10^{-3}$ ) to the $\mathrm{MeV}$ one (associated to $\mathrm{q}=1$ ). The horizontal axis deploys the real part of the momentum $p$, that we call $x$. The thick curves correspond to $q=1$ and the thin ones to $q>1$. Curves for three values of $y=\mathcal{I}(p)$ are drawn in each figure. Red 


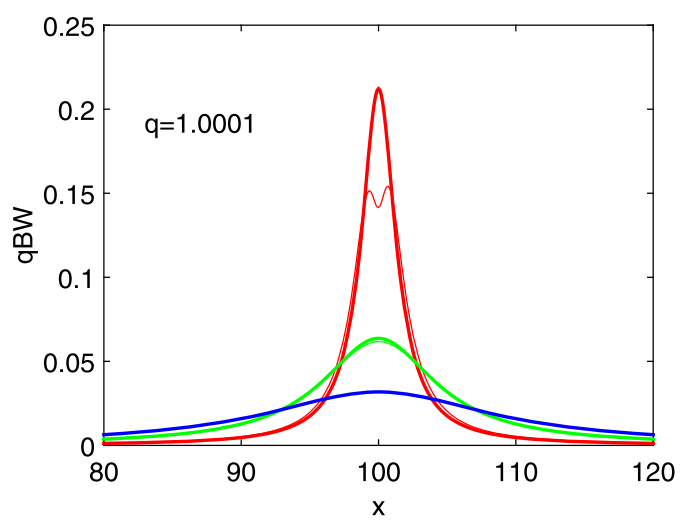

Fig. 2. $q B W$ and orthodox BW vs. $x$, the real part of $p$, for $q=1+10^{-4}$. For additional details, see text. (For interpretation of the references to color in this figure, the reader is referred to the web version of this article.)

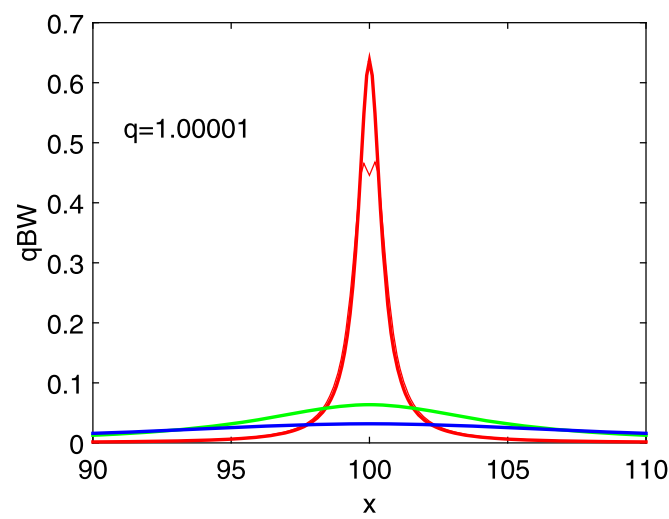

Fig. 3. $\mathrm{qBW}$ and orthodox BW vs. $x$, the real part of $p$, for $q=1+10^{-5}$. For additional details, see text. (For interpretation of the references to color in this figure, the reader is referred to the web version of this article.)

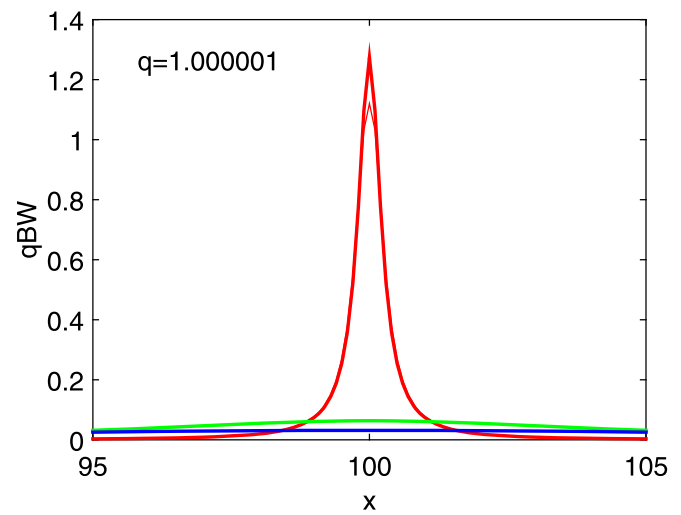

Fig. 4. $\mathrm{qBW}$ and orthodox BW vs. $x$, the real part of $p$, for $q=1+10^{-6}$. For additional details, see text. (For interpretation of the references to color in this figure, the reader is referred to the web version of this article.) 
for $y=0.25$, green for $y=5$, and blue for $y=10$. One appreciates the fact that a) for smaller $y$ 's the qBW differs in a more significant fashion from the orthodox BW values and $b$ ) these differences grow as the energy augments. In this paper we are interested in small-q resonances. We see that they should be seen at energies ranging from the $\mathrm{MeV}$ to the $\mathrm{GeV}$ scales.

\section{Conclusions}

It is the essence to point out that, as discussed in [2], empirically one often obtains q-Gaussians rather than pure Gaussians, with q very close to one. Accordingly, for a q-region in the immediate neighborhood of $\mathrm{q}=1$ we have here studied the main properties of the associated q-Gamow states, that are solutions to the NRT-nonlinear, q-generalization of Schroedinger's equation [21, 22].

We have computed their norm, the mean energy value, and the concomitant q-Breit-Wigner distributions. In all instances, results tend to the customary ones when the all important q-parameter of Tsallis' obeys $q \rightarrow 1$. Accordingly, in this effort we introduced new intermediate energy q-Gamow states.

Our main result is that our q-Breit-Wigner probability distribution will differ from the usual one according to the factor 3.4, which might be checked out, after careful error's analysis, in extant accelerator data, thus proving the existence of the new q-Gamow states we are advancing here.

\section{Acknowledgement}

The authors thank CONICET grant PIP029/12.

\section{Appendix A}

\section{A.1. Review on Gamow states}

This appendix summarizes results from [18].

A Gamow state, at large distances from the scattering center has the form

$$
\left|\psi_{\mathrm{G}}\right\rangle=\int_{-\infty}^{\infty}\{\mathcal{H}[\mathfrak{I}(p)] \mathcal{H}(x)-\mathcal{H}[-\mathfrak{I}(p)] \mathcal{H}(-x)\} e^{\frac{i p x}{\hbar}}|x\rangle \mathrm{d} x .
$$

The square of the norm reads

$$
\left\langle\psi_{\mathrm{G}} \mid \psi_{\mathrm{G}}\right\rangle=\int_{0}^{\infty} \mathcal{H}[\Im(p)] e^{\frac{i\left(p-p^{*}\right) x}{\hbar}} \mathrm{d} x-\int_{-\infty}^{0} \mathcal{H}[-\mathfrak{I}(p)] e^{\frac{i\left(p-p^{*}\right) x}{\hbar}} \mathrm{d} x .
$$

These integrals can be easily evaluated. One finds

$$
\left\langle\psi_{\mathrm{G}} \mid \psi_{\mathrm{G}}\right\rangle=\{\mathcal{H}[\mathfrak{I}(p)]-\mathcal{H}[-\mathfrak{I}(p)]\} \frac{\hbar}{i\left(p^{*}-p\right)}=\frac{\hbar}{2|\mathfrak{I}(p)|} .
$$

Accordingly, the normalized Gamow-state $\phi_{\mathrm{G}}$ becomes [10]

$$
\left|\phi_{G}\right\rangle=\sqrt{\frac{2|\mathfrak{I}(p)|}{\hbar}}\left|\psi_{G}\right\rangle .
$$


Additionally,

$$
\begin{aligned}
\left\langle\phi_{\mathrm{G}}\right|\left(\mathrm{H}\left|\phi_{\mathrm{G}}\right\rangle\right) & =\frac{\mathrm{p}^{2}}{2 \mathrm{~m}}, \\
\left(\left\langle\phi_{\mathrm{G}}\right| \mathrm{H}\right)\left|\phi_{\mathrm{G}}\right\rangle & =\frac{\mathrm{p}^{* 2}}{2 \mathrm{~m}},
\end{aligned}
$$

The mean energy is

$$
\langle\mathrm{H}\rangle=\frac{1}{2}\left[\left\langle\phi_{\mathrm{G}}\right|\left(\mathrm{H}\left|\phi_{\mathrm{G}}\right\rangle\right)+\left(\left\langle\phi_{\mathrm{G}}\right| \mathrm{H}\right)\left|\phi_{\mathrm{G}}\right\rangle\right]=\frac{\mathrm{p}^{2}+\mathrm{p}^{* 2}}{4 \mathrm{~m}}=\frac{\mathfrak{R}\left(\mathrm{p}^{2}\right)}{2 \mathrm{~m}} .
$$

In order to obtain de probability distribution associated to a Gamow state we start by the looking at the scalar product between this state and a free one

$$
\left\langle\phi \mid \phi_{\mathrm{G}}\right\rangle=\frac{1}{\hbar} \sqrt{\frac{|\mathfrak{I}(\mathrm{p})|}{\pi}}\left\{\int_{0}^{\infty} \mathcal{H}[\mathfrak{I}(p)] e^{\frac{i(p-k) x}{\hbar}} \mathrm{d} x-\int_{-\infty}^{0} \mathcal{H}[-\mathfrak{I}(p)] e^{\frac{i(p-k) x}{\hbar}} \mathrm{d} x\right\} .
$$

Thus,

$$
\left\langle\phi \mid \phi_{G}\right\rangle=\frac{i \sqrt{\frac{|\Im(p)|}{\pi}}}{p-k} .
$$

The ensuing probability distribution is the Breit-Wigner one [10]

$$
\left|\left\langle\phi \mid \phi_{G}\right\rangle\right|^{2}=\frac{|\mathfrak{I}(p)|}{\pi\left\{[\mathfrak{R}(p)-k]^{2}+\mathfrak{I}(p)^{2}\right\}} .
$$

\section{References}

[1] N. Boccara, Modeling Complex Systems, Springer, Berlin, 2004.

[2] C. Vignat, A. Plastino, Physica A 388 (2009) 601.

[3] M. Scroeder, Fractals, Chaos, Power Laws, Freeman, NY, 1991.

[4] C. Tsallis, Introduction to Nonextensive Statistical Mechanics, Springer, Berlin, 2009.

[5] C.G. Bollini, O. Civitarese, A.L. De Paoli, M.C. Rocca, Phys. Lett. B 382 (1996) 205.

[6] C.G. Bollini, O. Civitarese, A.L. De Paoli, M.C. Rocca, J. Math. Phys. 37 (1996) 4235.

[7] C.G. Bollini, O. Civitarese, A.L. De Paoli, M.C. Rocca, Gamow states in a rigged Hilbert space, in: A. Bohm, H.D. Doebner, P. Kielanowski (Eds.), Irreversibility and Causality. Semigroups and Rigged Hilbert Spaces, in: Lecture Notes in Physics, vol. 504, Springer-Verlag, 1998, p. 34. Proceedings of the Symposium on Semigroups at the 21st Colloquium on Group Theoretical Methods in Physics - Group 21 -, Goslar, July 15-19 (1996).

[8] O. Civitarese, A.L. De Paoli, M.C. Rocca, Nucl. Phys. A 642 (1998) 531.

[9] G. Gamow, Z. Phys. 51 (1928) 204;

G. Gamow, Z. Phys. 52 (1928) 510.

[10] T. Berggren, Nucl. Phys. A 109 (1968) 265.

[11] J. Sebastiao e Silva, Math. Ann. 136 (1958) 38.

[12] M. Hasumi, Tohoku Math. J. 13 (1961) 94.

[13] M. Morimoto, Proc. Jpn. Acad. 51 (1975) 87;

M. Morimoto, Proc. Jpn. Acad. 51 (1975) 213.

[14] I.M. Guelfand, N.Y. Vilenkin, Les Distributions, Tome IV, Dunod, Paris, 1967.

[15] A. Bohm, J. Math. Phys. 21 (1980) 1040;

A. Bohm, J. Math. Phys. 22 (1981) 2813. 
[16] M. Gadella, J. Math. Phys. 24 (1983) 1462;

M. Gadella, J. Math. Phys. 24 (1983) 2142;

M. Gadella, J. Math. Phys. 25 (1984) 2481.

[17] R. de la Madrid, Nucl. Phys. A 812 (2008) 13;

R. de la Madrid, Nucl. Phys. A 940 (2015) 297.

[18] A. Plastino, M.C. Rocca, Nucl. Phys. A 948 (2016) 19.

[19] F. Barile, et al., ALICE Collaboration, EPJ Web Conf. 60 (2013) 13012;

B. Abelev, et al., ALICE Collaboration, Phys. Rev. Lett. 111 (2013) 222301;

Yu.V. Kharlov, ALICE Collaboration, Phys. At. Nucl. 76 (2013) 1497;

ALICE Collaboration, Phys. Rev. C 91 (2015) 024609;

ATLAS Collaboration, New J. Phys. 13 (2011) 053033;

CMS Collaboration, J. High Energy Phys. 05 (2011) 064;

CMS Collaboration, Eur. Phys. J. C 74 (2014) 2847.

[20] A. Adare, et al., PHENIX Collaboration, Phys. Rev. D 83 (2011) 052004;

PHENIX Collaboration, Phys. Rev. C 83 (2011) 024909;

PHENIX Collaboration, Phys. Rev. C 83 (2011) 064903;

PHENIX Collaboration, Phys. Rev. C 84 (2011) 044902.

[21] F.D. Nobre, M.A. Rego-Monteiro, C. Tsallis, Phys. Rev. Lett. 106 (2011) 140601;

A.R. Plastino, A.M.C. Souza, F.D. Nobre, C. Tsallis, Phys. Rev. A 90 (2014) 062134;

A.R. Plastino, C. Tsallis, J. Math. Phys. 54 (2013) 041505;

S. Curilef, A.R. Plastino, A. Plastino, Physica A 392 (2013) 2631;

I.V. Toranzo, A.R. Plastino, J.S. Dehesa, A. Plastino, Physica A 392 (2013) 3945.

[22] A. Plastino, M.C. Rocca, Phys. Lett. A 379 (2015) 2690.

[23] N. Michel, W. Nazarewicz, M. Ploszajczak, T. Vertse, J. Phys. G 36 (2009) 013101. 